\title{
Electronic structure and x-ray magnetic circular dichroism of Mn-doped $\mathrm{TiO}_{2}$
}

\author{
L.V. Bekenov and V.N. Antonov \\ G.V. Kurdyumov Institute for Metal Physics National of the Sciences of Ukraine \\ 36 Academician Vernadsky Blvd., UA-03680, Kiev-142, Ukraine \\ E-mail: vantonov@mpi-halle.mpg.de
}

Max-Planck Institut für Mikrostrukturphysik, Weinberg 2, D-06120 Halle

Received May 8, 2015, published online October 23, 2015

\begin{abstract}
The electronic structure of (Ti,Mn) $\mathrm{O}_{2}$ diluted magnetic semiconductors was investigated theoretically from first principles using the fully relativistic Dirac linear muffin-tin orbital band structure method. The electronic structure was obtained with the local spin-density approximation taking into account strong Coulomb correlations in the frame of the LSDA $+U$ approximation. The $x$-ray absorption spectra and x-ray magnetic circular dichroism spectra at the $\mathrm{Mn}$ and $\mathrm{Ti} L_{2,3}$ and $\mathrm{O} K$ edges were investigated theoretically from first principles. The origin of the XMCD spectra in these compounds was examined. The calculated results are compared with available experimental data.
\end{abstract}

\section{PACS: 75.50.Cc Other ferromagnetic metals and alloys; \\ 71.20.Lp Intermetallic compounds; \\ 71.15.Rf Relativistic effects.}

Keywords: electronic structure, spintronics, magnetic semiconductors.

\section{Introduction}

Spintronics or spin-transport electronics has attracted much of attention because of its technologically potential applications. Diluted magnetic semiconductors (DMSs), obtained by inserting a magnetic element as impurity into the host semiconductor, can be used for the spintronic devices [1]. The DMSs are among the most intensely investigated materials in recent times in view of their great application potential. Yet, they are also the most controversial because of the possibility of extrinsic effects attributable to dopant solubility and clustering, point defects, incorporation of unintentional impurities, etc. The starting materials, which were expected to be the promising candidates for spintronics, are group III-V materials, such as (Ga,Mn)As [2,3] with the highest Curie temperature of $\sim 110 \mathrm{~K}$ [4]. Other candidates, which can show this property, are transition metal (TM) doped Group III nitrides, phosphides and semiconducting oxides. Dietl et al. [5] predicted theoretically a Curie temperature $T_{C}$ higher than room temperature for transition element doped semiconducting materials, such as, $\mathrm{GaN}, \mathrm{TiO}_{2}, \mathrm{SnO}_{2}$, and $\mathrm{ZnO}$.

As a wide-band-gap semiconductor, $\mathrm{TiO}_{2}(3.2 \mathrm{eV}$ for the anatase phase and $3.0 \mathrm{eV}$ for the rutile phase) has been considered as a base material for realizing transparent DMSs. The transition-metal-doped ( $\mathrm{V}, \mathrm{Cr}, \mathrm{Fe}, \mathrm{Ni}$, etc.) $\mathrm{TiO}_{2}$ alloys have been under numerous experimental and theoretical investigations [6-12] on their magnetic properties and the related structural and electronic properties since the discovery of room-temperature ferromagnetism in Co-doped anatase $\mathrm{TiO}_{2}$ thin films [13]. Despite the intensive research on $\mathrm{TiO}_{2}$-based magnetic oxides, the interpretations for the observed ferromagnetic (FM) properties have often been controversial, e.g., on whether the exhibited ferromagnetism is intrinsic or not [12]. For spintronic applications the ferromagnetism in a semiconductor needs to be intrinsic, that is, not from magnetic clusters of the doped transition-metal impurities. Some studies suggest that the FM in TM-doped $\mathrm{TiO}_{2}$ originates from the presence of secondary phases or FM clusters, whereas other results indicate the existence of intrinsic FM of TM substitution in the $\mathrm{TiO}_{2}$ lattice. Xu et al. [14] reported room temperature FM in $\mathrm{Mn}$-doped $\mathrm{TiO}_{2}$ and claimed its origin to be intrinsic. This group explained that FM is due to the coupling between the Mn magnetic moments via large concentration of holes, whereas, Hong et al. [15] argued that Mn doping did not play any role in inducing the FM. Also, a number of recent studies showed that the observed 
ferromagnetism tends to depend on methods and conditions used in the sample preparation. Thus, no consensus on the proper origin of the room-temperature ferromagnetism in such metal-oxide semiconductors has been reached yet. Therefore, more intensive and extensive study is essential.

The electronic and magnetic properties of TM-doped $\mathrm{TiO}_{2}$ have been calculated from first principles using a wide range of different methods [16-27]. Most interest in previous investigations was concentrated on the nature of the magnetic interactions in the DMSs. In the present study, we focus our attention on the x-ray absorption spectra (XAS) as well as x-ray magnetic circular dichroism (XMCD) in Mn-doped $\mathrm{TiO}_{2}$. XMCD experiments measure the difference of the absorption of $\mathrm{x}$-rays with opposite (left and right) directions of circular polarization. The XMCD is a powerful tool to study the element-specific local magnetic interactions and also it reflects the spin and orbital polarizations of the local electronic states. The XAS and XMCD spectra in the Mn-, Fe-, and Co-doped $\mathrm{TiO}_{2}$ were measured by several groups [11,28-31].

The paper is organized as follows. Section 2 presents structural models of $(\mathrm{Ti}, \mathrm{Mn}) \mathrm{O}_{2}$ DMSs and the details of the calculations. Section 3 is devoted to the electronic structure as well as XAS and XMCD properties of Mn-doped $\mathrm{TiO}_{2}$ calculated with the fully relativistic Dirac LMTO band structure method. The results are compared with available experimental data. Finally, the results are summarized in Sec. 4.

\section{Computational details}

\subsection{Crystal structure}

$\mathrm{TiO}_{2}$ has three of the most commonly encountered crystalline polymorphs: anatase, brookite, and rutile. All the three crystal structures are made up of distorted $\mathrm{TiO}_{6}$ octahedra, but in different ways [32]. Among the polymorphs of $\mathrm{TiO}_{2}$, the rutile phase exhibits an excellent combination of physical properties such as exceptional light scattering efficiency, high refractive index, opacity, chemical inertness, and superb photocatalytic properties. Rutile $\mathrm{TiO}_{2}$ can be obtained via the high-temperature calcination of anatase nanoparticles; however, calcination unavoidably leads to agglomeration and growth of the nanocrystallites. So far, many groups have carried out significant work to explore novel methods to prepare nanocrystalline rutile $\mathrm{TiO}_{2}$ by controlling the size and morphology as well as related properties [33,34].

The vast majority of studies of the bulk and surface properties of $\mathrm{TiO}_{2}$ have been for the rutile phase. The rutile structure, illustrated in Fig. 1, belongs to the $P 4_{2} / \mathrm{mnm}$ tetragonal space group (number 136). The unit cell is defined by the lattice vectors $\mathbf{a}$ and $\mathbf{c}$ and contains two $\mathrm{TiO}_{2}$ units with Ti ions at $(0,0,0)$ and $(1 / 2,1 / 2,1 / 2)$, and $O$ ions at $\pm(u, u, 0)$ and $\pm(u+1 / 2,1 / 2-u, 1 / 2)$. The unit-cell

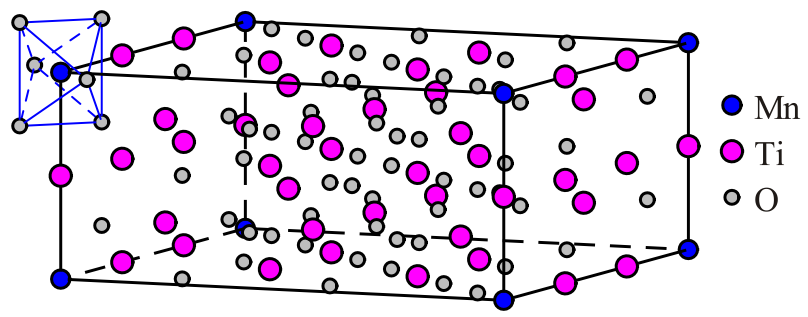

Fig. 1. (Color online) Schematic representation of the $3 a \times 3 a \times 2 c$ supercell of the rutile-type $\mathrm{TiO}_{2}$ unit cell with one of the Ti ions replaced by Mn one.

parameters are found to be $a=4.6019 \AA, c=2.9974 \AA$, and $u=0.30392$ at $15 \mathrm{~K}$ [35]. Each Ti ion is octahedrally coordinated to six oxygen ions. The $\mathrm{TiO}_{2}$ octahedron is distorted, with the apical Ti-O bond length (1.9779 $\AA$ ) being slightly longer than the equatorial $\mathrm{Ti}-\mathrm{O}$ bond length (1.9684 $\AA$ ). The four equatorial O ions are coplanar occupying a rectangular arrangement with the long edge along the $c$ direction and the short edge lying diagonally across the plane defined by the $a$ direction. The $\mathrm{TiO}_{6}$ octahedra form chains that share edges along the $c$ direction and share vertices in the $a-b$ plane.

The calculations of the electronic structure of the $(\mathrm{Ti}, \mathrm{Mn}) \mathrm{O}_{2}$ DMSs were performed for $3 a \times 3 a \times 1 c$ and $3 a \times 3 a \times 2 c$ supercells of the rutile-type $\mathrm{TiO}_{2}$ unit cell with one of the Ti ions replaced by Mn. The super-cell calculations were performed for the compositions $x=0.067(1 / 15)$ and $x=0.037$ (1/27) using the simple monoclinic $P 12 / \mathrm{m} 1$ (No. 10) space group. The substitutional $\left(\mathrm{Ti}_{1-\chi} \mathrm{Mn}_{\chi}\right) \mathrm{O}_{2}$ positions are illustrated in Fig. 1 for a 76-atom $\mathrm{TiO}_{2}$ unit cell containing one substitutional $\mathrm{Mn}$ atom $(x=0.037)$. The Mn atom has six oxygen nearest neighbors: four $\mathrm{O}$ atoms at the distance of $1.9684 \AA$ and two $O$ atoms at $1.9779 \AA$. The second-neighbor shell consists of $10 \mathrm{Ti}$ atoms: two at the distance of $2.9974 \AA$, and eight at $3.5826 \AA$.

\subsection{Computational details}

The details of the computational method are described in our previous papers [36-38], and here we only mention some aspects specific to the present calculations. The calculations were performed using the spin-polarized fully relativistic linear-muffin-tin-orbital (SPR LMTO) method $[39,40]$ for the experimentally observed lattice constants: $a=4.6019 \AA, c=2.9974 \AA$ for rutile-type $\mathrm{TiO}_{2}$ [35]. We used the Perdew-Wang [41] parameterization of the exchange-correlation potential. The Brillouin zone (BZ) integrations were performed using the improved tetrahedron method [42], and the charge self-consistency was obtained on a grid of $170 \mathbf{k}$ points in the irreducible part of the (Ti,Mn) $\mathrm{O}_{2}$ BZ. To improve the potential we included additional interstitial spheres. The basis consisted of Ti and $\mathrm{Mn}$ $s, p, d$ and $f, \mathrm{O} s, p$ and $d$, and empty spheres $s$ and $p$ LMTOs. 
The x-ray absorption and dichroism spectra were calculated taking into account the exchange splitting of core levels. The finite lifetime of a core hole was accounted for by folding the spectra with a Lorentzian. The widths of $L_{2}$ and $L_{3}$ core level spectra were taken from Ref. 43: $\Gamma_{L_{2}}=$ $=0.52 \mathrm{eV}, \Gamma_{L_{3}}=0.25 \mathrm{eV}$ for Ti; $\Gamma_{L_{2}}=0.97 \mathrm{eV}, \Gamma_{L_{3}}$ $=0.36 \mathrm{eV}$ for $\mathrm{Mn}$; and $\Gamma_{K}=0.18 \mathrm{eV}$ for $\mathrm{O}$. The finite apparative resolution of the spectrometer was accounted for by a Gaussian of width $0.6 \mathrm{eV}$.

Since the local density approximation fails to describe correctly strongly localized $\mathrm{Mn} d$ states in (Ti,Mn) $\mathrm{O}_{2}$, we treated these states within the LSDA $+U$ approach [44]. We used the rotationally invariant LSDA $+U$ method described in detail in our previous paper [45]. For the exchange integral $J$ the value of $0.95 \mathrm{eV}$ estimated from constrained LSDA calculations was used. The effective onsite Coulomb repulsion $U$ was considered as an adjustable parameter. We found that the best agreement between the theory and the experiment on the XAS and XMCD spectra was achieved for $U=4 \mathrm{eV}$. These values of $U$ and $J$ parameters were fixed and used for all the calculations presented in the paper.

\section{Results and discussion}

\subsection{Energy band structure}

Figure 2 presents the total and partial density of states (DOS) for a 76-atom $\mathrm{TiO}_{2}$ rutile-type unit cell containing one $\mathrm{Mn}_{\mathrm{Ti}}$ substitution $(x=0.037)$ obtained in the LSDA $+U$ approximation for a ferromagnetic arrangement of the $\mathrm{Mn}$ moments. The $\mathrm{O} 2 \mathrm{~s}$ states are located mostly between -17.6 and $-15.8 \mathrm{eV}$ below the Fermi level (not shown) and the $2 p$ states of $\mathrm{O}$ are found from $-5.6 \mathrm{eV}$ to the $E_{F}$ and from 2.5 to $8 \mathrm{eV}$ above the Fermi level. The spin splitting of the $\mathrm{O} p$ states is quite small. The Ti $3 d$ states occupy the energy interval between -5.4 and $-0.2 \mathrm{eV}$ and hybridize strongly with the oxygen $2 p$ states. The empty Ti $3 d$ states form two groups of energy bands with an energy gap in between. The first group is located between $2.35 \mathrm{eV}$ and $4.6 \mathrm{eV}$, the second one is between 5.1 and $7.9 \mathrm{eV}$. The second group is mostly of $t_{2 g}$ symmetry, the first group is of $e_{g}$ symmetry, but because of rather low symmetry $(P 12 / m 1)$ has significant amount of the $t_{2 g}$ states.

The crystal field at the $\mathrm{Mn}_{\mathrm{Ti}}$ site $\left(C_{2 h}\right.$ point symmetry) causes the splitting of $\mathrm{Mn} d$ orbitals into five singlets: $a_{g 1}\left(d_{3 z-1}\right), a_{g 2}\left(d_{x^{2}-y^{2}}\right), a_{g 3}\left(d_{x y}\right), b_{g 1}\left(d_{x z}\right), b_{g 2}\left(d_{y z}\right)$. The Mn $3 d$ impurity states hybridize well with the $\mathrm{O} 2 p$ conduction band. The $d_{z x}^{\uparrow}+d_{y z}^{\uparrow}$ and $d_{x y}^{\uparrow}$ states are situated in the energy gap. The $d_{x^{2}-y^{2}}^{\downarrow}$ states are slightly hybridized with the bottom of empty Ti $3 e_{g}$ states, the $d_{z x}^{\downarrow}+d_{y z}^{\downarrow}$ states cross the top of $\mathrm{Ti} 3 e_{g}$ states. As a result, the Mn $3 d$

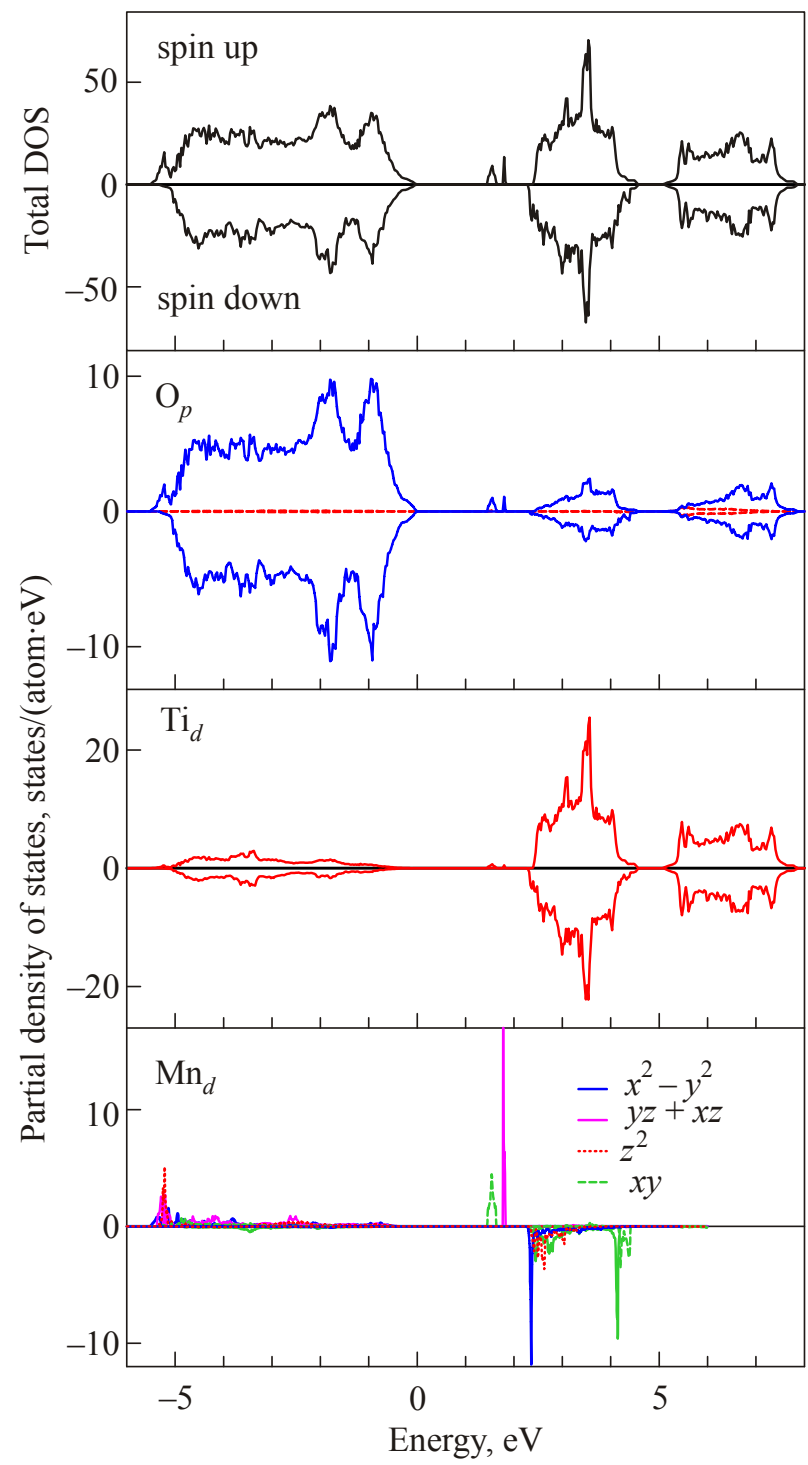

Fig. 2. (Color online) Total [in states/(cell·eV)] and partial [in states/(atom·eV)] densities of states for the $\mathrm{O}$, Ti and substitutional $\mathrm{Mn}_{\mathrm{Ti}}$ ions in $\left(\mathrm{Ti}_{1-x} \mathrm{Mn}_{x}\right) \mathrm{O}_{2}(x=0.0667)$. The Fermi energy is at zero.

empty states consist of very narrow peaks with weak Mn 3d-Ti 3d hybridization. The picture of this kind indicates the correlation nature of $\mathrm{Mn} 3 d$ states in doped $\mathrm{TiO}_{2}$ DMSs. There is a double peak structure in the oxygen $2 p$ partial DOS in the energy gap because of the Mn $3 d-\mathrm{O} 2 p$ hybridization.

Our band structure calculations yield the spin magnetic moment of $3.039 \mu_{B}$ for the $\mathrm{Mn}$ atoms in the $\left(\mathrm{Ti}_{1-x} \mathrm{Mn}_{x}\right) \mathrm{O}_{2}$. The induced spin magnetic moments at the $\mathrm{O}$ first neighbor sites are of $-0.002 \mu_{B}$ and $-0.022 \mu_{B}$ for longer and shorter distant $\mathrm{O}$ atoms, respectively. Ten $\mathrm{Ti}$ ions in the second neighbor shell couple ferromagnetically to the substitutional $\mathrm{Mn}$ ion with the spin magnetic moments from $0.002 \mu_{B}$ to $0.011 \mu_{B}$. The orbital magnetic moments at the Ti and $\mathrm{O}$ sites are small with the largest one at the $O$ first neighbor 
sites $\left(-0.003 \mu_{B}\right)$. The orbital magnetic moment at the Mn site is $-0.012 \mu_{B}$ and is opposite to the spin moment.

\subsection{X-ray absorption and XMCD spectra at the Ti and Mn $L_{2,3}$ edges}

The XAS and XMCD spectra at the Ti, Mn $L_{2,3}$ and O $K$ edges in the Mn-doped $\mathrm{TiO}_{2}$ were measured by several groups [11,28-31]. Figure 3 presents experimental XAS and XMCD spectra of (Ti,Mn)O $\mathrm{O}_{2}$ at the $\mathrm{Mn} L_{2,3}$ edges [29] together with the spectra calculated in the LSDA $+U$ approximation. The experimentally measured $\mathrm{Mn} L_{2,3}$ XAS exhibit spectral features in the ranges of $635-640 \mathrm{eV}$ and 645-650 eV. These spectral features were assigned to $\mathrm{Mn}$ $2 p_{3 / 2} \rightarrow 3 d_{3 / 2,5 / 2}\left(L_{3}\right)$ and Mn $2 p_{1 / 2} \rightarrow 3 d_{3 / 2}\left(L_{2}\right)$ transitions, respectively [29]. The X-ray absorption spectrum at the $\mathrm{Mn} L_{3}$ edge is rather complicated and consists of a major structure at $636.3 \mathrm{eV}$ and two high energy shoulders at $637.8 \mathrm{eV}$ and $640 \mathrm{eV}$. The theory reproduces the energy position and intensity of the major peak and shoulder at $637.8 \mathrm{eV}$ quite well but fails to describe the high energy shoulder at $640 \mathrm{eV}$, which probably has satellite nature. The Mn $L_{2}$ XAS has a double peak structure. The theory reproduces the peaks but with inverse relative intensity.

Kumar et al. [29] discovered that the line shape of the spectral features for the Mn-doped $\mathrm{TiO}_{2}$ films are different from $\mathrm{MnO}_{2}$ [24], $\mathrm{Mn}_{2} \mathrm{O}_{3}$ [25], and $\mathrm{Mn}$ metal [29]. On the other hand, the line shape of the spectral features looks similar to $\mathrm{MnO}$ and $\mathrm{MnS}$ [24,27]. On the basis of these observations Kumar et al. [29] concluded that the Mn ions are in the $2+$ valence state in the $\mathrm{TiO}_{2}$ matrix. It is in

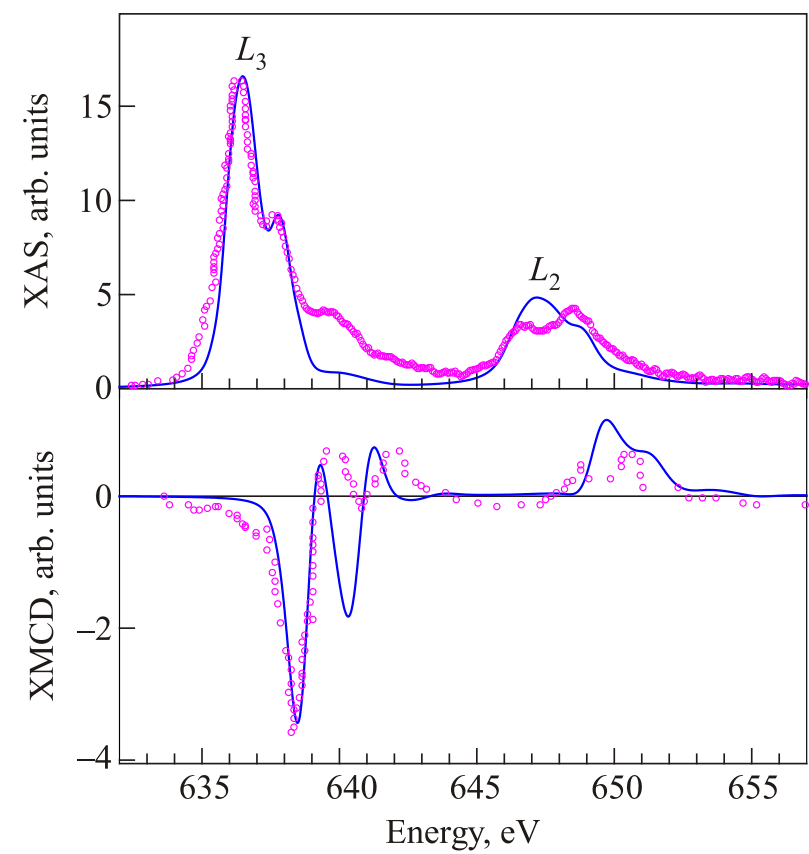

Fig. 3. (Color online) X-ray absorption (top panel) and XMCD (lower panel) experimental spectra (circles) [29] of $\left(\mathrm{Ti}_{1-x} \mathrm{Mn}_{x}\right) \mathrm{O}_{2}$ $(x=0.05)$ at the Mn $L_{2,3}$ edges and theoretically calculated spectra (full blue curves) for $x=0.0667$. agreement with our LSDA + $U$ calculations, which produce the Mn valency equal to $2.4+$.

The XMCD spectrum at the $M n L_{3}$ edge is also quite complicated and consists of a major peak with the negative sign and two positive high energy peaks. The theory reproduces the energy position and intensity of the major negative peak quite well. However, the theoretically calculated two high energy positive peaks are slightly shifted towards smaller energy in comparison with the experimental spectrum. The theory also produces a deeper minimum between the two high energy peaks than the experimental spectrum.

Figure 4 presents experimental XAS of $(\mathrm{Ti}, \mathrm{Mn}) \mathrm{O}_{2}$ at the Ti $L_{2,3}$ edges (upper panel) [29] together with the spectra calculated in the LSDA $+U$ approximation. The experimentally measured $\mathrm{Ti} L_{2,3}$ XAS consist of four major peaks in the range of 458-469 eV and a high energy shoulder at $471 \mathrm{eV}$. It is well known that the separation between the two main peaks in the $L_{3}$ (peaks $e_{g}$ and $t_{2 g}$ ) and $L_{2}$ (peaks $e_{g}$ and $t_{2 g}$ ) edges is associated with the crystalfield splitting modified by the exchange interaction. Because the spin-orbit (SO) splitting of the core Ti $2 p$ level $(5.74 \mathrm{eV})$ and the Ti $3 d$ crystal-field splitting modified by the exchange interaction $(3.3 \mathrm{eV})$ are of the same order of magnitude, the $L_{3}$ and $L_{2}$ x-ray absorption spectra are strongly overlapped. The four experimentally observed intense peaks from 456 to $466 \mathrm{eV}$ can, to a first approximation, be assigned to $2 p_{3 / 2} \rightarrow e_{g}, 2 p_{3 / 2} \rightarrow t_{2 g}, 2 p_{1 / 2} \rightarrow e_{g}$, and

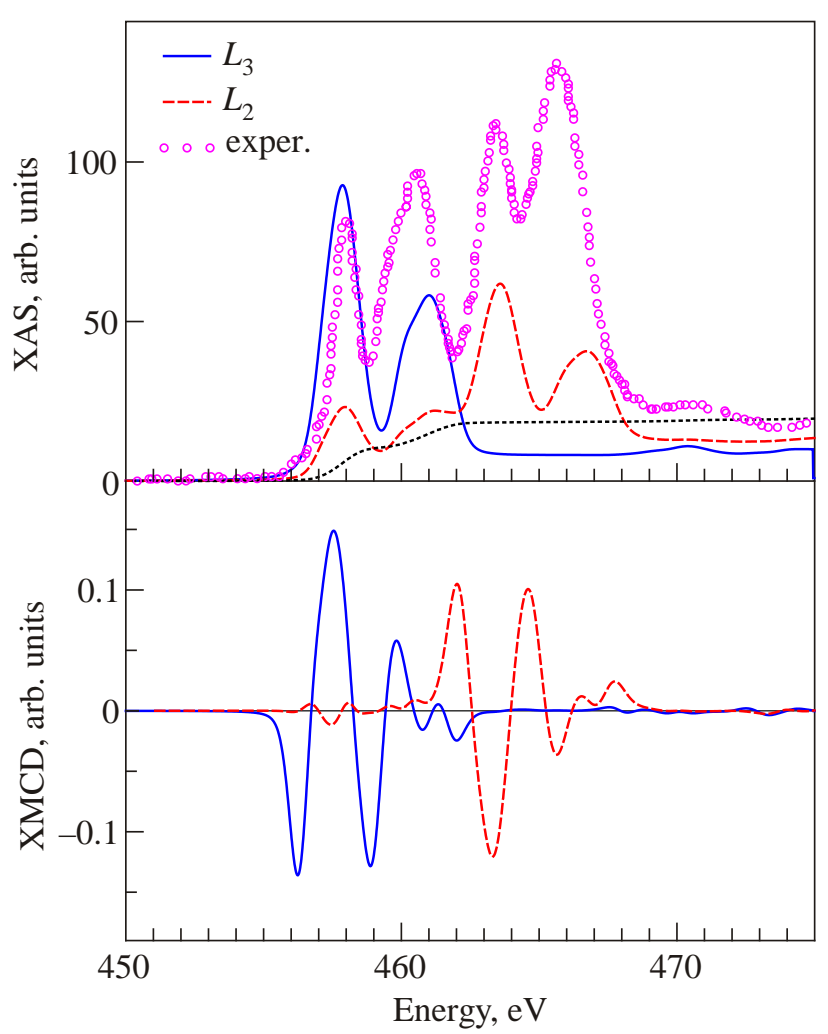

Fig. 4. (Color online) X-ray absorption (top panel) and XMCD (lower panel) experimental spectra (circles) [29] of $\left(\mathrm{Ti}_{1-x} \mathrm{Mn}_{x}\right) \mathrm{O}_{2}$ $(x=0.05)$ at the $\mathrm{Ti} L_{2,3}$ edges and theoretically calculated spectra. 
$2 p_{1 / 2} \rightarrow t_{2 g}$ transitions, respectively. However, as can be clearly seen from Fig. 3 (upper panel), the $2 p_{1 / 2} \rightarrow e_{g}$ and $2 p_{1 / 2} \rightarrow t_{2 g}$ transitions ( $L_{2}$ spectrum) contribute also to the two low energy peaks. The theory reproduces the energy position of all the fine structures quite well, however, does not produce the experimentally observed $L_{3} / L_{2} \mathrm{X}$-ray absorption ratio. It is well known that the $L_{2}$ and $L_{3}$ absorption channels in early $3 d$ transition metals with nearly empty $d$ bands are strongly coupled through the photoelectron-core-hole Coulomb and exchange interactions [46-49]. This leads to a branching ratio close to $1: 1$, far from the statistical ratio 2:1, which is obtained in the single-particle theory, unless the SO interaction in the final $3 d$ band is considered. From our band structure calculations we obtained the $L_{3} / L_{2}$ branching ratio equal to 1.65 which is far from the experimentally observed. This problem can be accounted for through many-electron calculations and we address it for future investigations.

The XMCD spectra at the Ti $L_{3}$ and $L_{2}$ edges are well pronounced and show quite complicated shapes with several negative and positive peaks. The experimental measurements of the XMCD spectra at Ti $L_{2,3}$ edges are highly desirable.

\section{3. $X$-ray absorption spectra at the $O \mathrm{~K}$ edge}

The XAS and XMCD spectra in metals at the $K$ edge where the $1 \mathrm{~s}$ core electrons are excited to the $p$ states through the dipolar transition usually attract only minor interest because $p$ states are not the states that influence the magnetic and orbital orders. Recently, however, the understanding of $p$ states has become important since XMCD spectroscopy using the $K$ edges of transition metals became popular. Because of the delocalized nature of $p$ states, the $K$ edge XMCD is sensitive to the electronic structure of neighboring sites.

The x-ray absorption spectra at the oxygen $K$ edge in the $\left(\mathrm{Ti}_{1-x} \mathrm{Mn}_{x}\right) \mathrm{O}_{3}(x=0.03$ and 0.05$)$ were measured by Kumar et al. [29]. The $O K$ edge spectra fundamentally reveal a transition from the $\mathrm{O} 1 \mathrm{~s}$ core state to the unoccupied $\mathrm{O} 2 p$ derived states, which are hybridized with the relatively narrow $3 d$ bands and broader $4 s p$ bands of the $\mathrm{Mn}$ and Ti ions. Figure 5 (upper panel) presents experimentally measured [29] O K x-ray absorption spectrum for $5 \% \mathrm{Mn}$-doped $\mathrm{TiO}_{2}$ in comparison with our calculations.

The O K XAS for Mn-doped $\mathrm{TiO}_{2}$ possess several fine structures such as a major double peak structure from 529 to $535 \mathrm{eV}$ with a low energy shoulder, and a wide structure between 538 and $550 \mathrm{eV}$. The latter spectral feature is due to the $\mathrm{O} 2 p$ orbitals hybridized with the Mn and Ti $4 s$ and $4 p$ orbitals. The small low energy shoulder at $528 \mathrm{eV}$ is attributed to the oxygen $2 p$ empty orbitals hybridized with the Mn $3 d$ orbitals situated in the energy gap (see Fig. 1). The major double peak occurring in the 529-535 eV energy range is attributed mainly to the oxygen $2 p$ empty orbitals hybridized with the Ti $3 d e_{g}$ and $t_{2 g}$ orbitals.

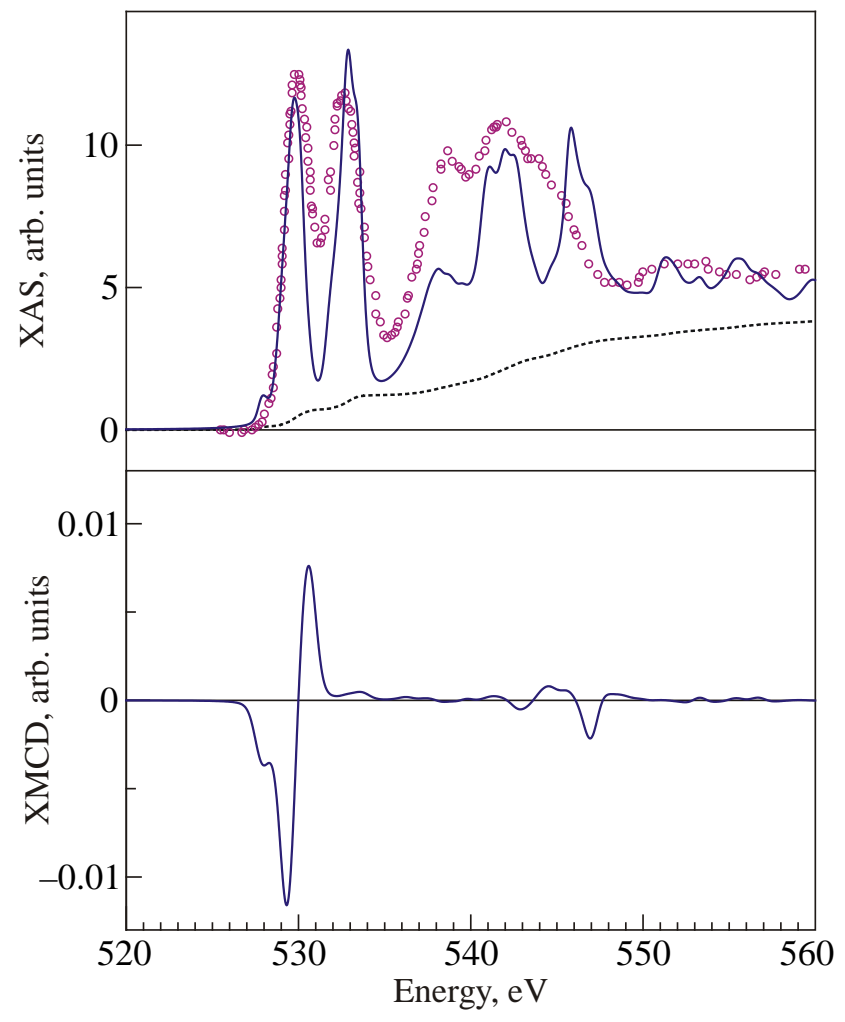

Fig. 5. (Color online) XAS (upper panel) and XMCD spectra (lower panel) at the $\mathrm{O} K$ edge in $\left(\mathrm{Ti}_{1-x} \mathrm{Mn}_{x}\right) \mathrm{O}_{2}(x=0.05)$ measured experimentally (circles) [29] and calculated theoretically (full blue curves).

The dichroism at the $\mathrm{O} K$ edge is significant only for the $2 p$ states that strongly hybridize with the Ti and Mn $3 d$ states in the energy range from 529 to $535 \mathrm{eV}$ (see Fig. 5 (lower panel)). The O K XMCD spectrum possesses an $S$-shape structure at this energy interval. The spectral XMCD features between 538 and $550 \mathrm{eV}$, which are due to the $\mathrm{O} 2 p$ orbitals hybridized with the $\mathrm{Mn}$ and Ti $4 s$ and $4 p$ orbitals, are relatively small.

\section{Summary}

We studied the electronic structure and $\mathrm{x}$-ray magnetic circular dichroism of $\mathrm{Mn}$-doped $\mathrm{TiO}_{2}$ diluted magnetic semiconductors by means of ab initio fully-relativistic spin-polarized Dirac linear muffin-tin orbital method. The x-ray absorption and dichroism spectra at the $\mathrm{Mn}$ and $\mathrm{Ti}$ $L_{2,3}$, and $\mathrm{O} K$ edges were investigated.

The Mn $3 d$ impurity states hybridize well with the $\mathrm{O} 2 p$ conduction band. The $d_{z x}^{\uparrow}+d_{y z}^{\uparrow}$ and $d_{x y}^{\uparrow}$ states are situated in the energy gap. The $d_{x^{2}-y^{2}}^{\downarrow}$ states are slightly hybridized with the bottom of empty Ti $e_{g}$ states, the $d_{z x}^{\downarrow}+d_{y z}^{\downarrow}$ states cross the top of Ti $3 e_{g}$ states. As a result, the Mn $3 d$ empty states consist of very narrow peaks with weak Mn $3 d-T i 3 d$ hybridization. The picture of this kind indicates the correlation nature of $\mathrm{Mn} 3 d$ states in doped $\mathrm{TiO}_{2}$ 
DMSs. There is a double peak structure in the oxygen $2 p$ partial DOS in the energy gap because of the Mn $3 d-\mathrm{O} 2 p$ hybridization. The empty Ti $3 d$ states are much wider than the Mn $3 d$ states and consist of two groups mostly of $t_{2 g}$ and $e_{g}$ symmetry. The theory reproduces the shape and energy positions of the major fine structures of the XAS and XMCD spectra at the Mn $L_{2,3}$ edge reasonably well. The Ti $L_{2,3}$ XAS consist of four major peaks and a high energy shoulder. The separation between the two main peaks in the $L_{3}$ (peaks $e_{g}$ and $t_{2 g}$ ) and $L_{2}$ (peaks $e_{g}$ and $t_{2 g}$ ) edges is associated with the crystal-field splitting modified by the exchange interaction. Because the spinorbit splitting of the core Ti $2 p$ level and the Ti $3 d$ crystalfield splitting are of the same order of magnitude the $L_{3}$ and $L_{2}$ x-ray absorption spectra are strongly overlapped. The four experimentally observed peaks from 456 to $466 \mathrm{eV}$ can be assigned to $2 p_{3 / 2} \rightarrow e_{g}, 2 p_{3 / 2} \rightarrow t_{2 g}$, $2 p_{1 / 2} \rightarrow e_{g}$, and $2 p_{1 / 2} \rightarrow t_{2 g}$ transitions, respectively. The theory reproduces the energy position of all the fine structures quite well, however, does not produce the experimentally observed $L_{3} / L_{2}$ x-ray absorption ratio. The $L_{2}$ and $L_{3}$ absorption channels in early $3 d$ transition metals with nearly empty $d$ bands are strongly coupled through the photoelectron-core-hole Coulomb and exchange interactions, which are not taken into account in present calculations.

The XAS and XMCD spectra at the oxygen $K$ edge reflect the electronic states of neighbouring sites because of the delocalized nature of $p$ states and strong O $2 p-\mathrm{Mn}, \mathrm{Ti}$ $3 d$ hybridization. The major double peak structure of the $\mathrm{O}$ $K$ XAS for Mn-doped $\mathrm{TiO}_{2}$ is attributed to the oxygen $2 p$ empty orbitals hybridized with the Ti $3 d e_{g}$ and $t_{2 g}$ orbitals. The wide structure between 538 and $550 \mathrm{eV}$ is due to the $\mathrm{O} 2 p$ orbitals hybridized with the Mn and Ti $4 s$ and $4 p$ orbitals. The small low energy shoulder at $528 \mathrm{eV}$ reflects the $\mathrm{O} 2 p-\mathrm{Mn} 3 d$ hybridization. The dichroism at the $\mathrm{O}$ $K$ edge is significant only for the $2 p$ states that strongly hybridize with the $\mathrm{Ti}$ and $\mathrm{Mn} 3 d$ states in the energy range from 529 to $535 \mathrm{eV}$. The spectral XMCD features between 538 and $550 \mathrm{eV}$, which are due to the $\mathrm{O} 2 p$ orbitals hybridized with the $\mathrm{Mn}$ and Ti $4 s$ and $4 p$ orbitals, are relatively small.

\section{Acknowledgments}

V.N.A. gratefully acknowledges the hospitality at MaxPlanck-Institut für Mikrostrukturphysik in Halle during his stay there.

1. J.K. Furdyna, Appl. Phys. Lett. 64, R 29 (1988).

2. S. Sanvito, G. Theurich, and N.A. Hill, J. Supercond. 15, 85 (2002).

3. T. Jungwirth, J. Sinova, J. Masek, J. Kucera, and A.H. MacDonald, Rev. Mod. Phys. 78, 809 (2006).
4. H. Ohno, A. Shen, F. Matsukura, A. Oiwa, A. Endo, S. Katsumoto, and Y. Iye, Appl. Phys. Lett. 69, 363 (1996).

5. T. Dietl, H. Ohno, F. Matsukura, J. Cibert, and D. Ferrand, Science 287, 1019 (2000).

6. W.K. Park, R.J. Ortega-Hertogs, J.S. Moodera, A. Punnoose, and M.S. Seehra, J. Appl. Phys. 91, 8093 (2002).

7. S.A. Chambers, T. Droubay, C.M. Wang, A.S. Leaa, R.F.C. Farrow, L. Folks, V. Deline, and S. Anders, Appl. Phys. Lett. 82, 1257 (2003).

8. Z. Wang, J. Tang, L. Tung, W. Zhou, and L. Spinu, J. Appl. Phys. 93, 7870 (2003).

9. N.H. Hong, J. Sakai, and A. Hassini, Appl. Phys. Lett. 84, 2602 (2004).

10. T. Droubay, S.M. Heald, V. Shutthanandan, S. Thevuthasan, S. Chambers, and J. Osterwalder, J. Appl. Phys. 97, 046103 (2005).

11. D.H. Kim, J.S. Yang, Y.S. Kim, T.W. Noh, S.D. Bu, S.-I. Baik, Y.-W. Kim, Y.D. Park, S.J. Pearton, J.-Y. Kim, J.-H. Park, H.-J. Lin, C.T. Chen, and Y.J. Song, Phys. Rev. B 71, 014440 (2005).

12. K.J. Kim, Y.R. Park, J.H. Lee, S. Choi, H.J. Lee, C.S. Kim, and J.Y. Park, J. Magn. Magn. Mater. 316, e215 (2007).

13. Y. Matsumoto, M. Murakami, T. Shono, T. Hasagawa, T. Fukumura, M. Kawasaki, P. Ahmet, T. Chikyow, S. Koshihara, and H. Koinuma, Science 291, 854 (2001).

14. J.P. Xu, J.F. Wang, Y.B. Lin, X.C. Liu, Z.L. Lu, Z.H. Lu, L.Y. Lv, F.M. Zhang, and Y.W. Du, J. Phys. D 40, 1278 (2007).

15. N.H. Hong, J. Sakai, A. Ruyter, and V. Brize, Appl. Phys. Lett. 89, 252504 (2006).

16. M.S. Park, S.K. Kwon, and B.I. Min, Phys. Rev. B 65, 161201(R) (2002).

17. M.S. Park and B.I. Min, Phys. Rev. B 68, 033202 (2003).

18. W.T. Geng and K.S. Kim, Phys. Rev. B 68, 125203 (2003).

19. H. Weng, X. Yang, J. Dong, H. Mizuseki, M. Kawasaki, and Y. Kawazoe, Phys. Rev. B 69, 125219 (2004).

20. G.Y. Gao, K.L. Yao, Z.L. Liu, Y.L. Li, J.L. Jiang, and Y.C. Li, Physica B 382, 14 (2006).

21. G.Y. Gao, K.L. Yao, and Z.L. Liu, Phys. Lett. A 359, 523 (2006).

22. R. Janisch and N.A. Spaldin, Phys. Rev. B 73, 035201 (2006).

23. M. Weissmanna and L.A. Errico, Physica B 398, 179 (2007).

24. R. Longa and N.J. Englisha, Appl. Phys. Lett. 98, 142103 (2011).

25. Q.L. Chen, B. Li, G. Zheng, K.H. He, and A.S. Zheng, Physica B 406, 3841 (2011).

26. L. Jia, C. Wu, S. Han, N. Yao, Y. Li, Z. Li, B. Chi, J. Pu, and L. Jian, J. Appl. Crystallogr. 509, 6067 (2011).

27. M. Khan, J. Xu, N. Chen, and W. Cao, J. Appl. Crystallogr. 513, 539 (2012).

28. J.-Y. Kim, J.-H. Park, B.-G. Park, H.-J. Noh, S.-J. Oh, J.S. Yang, D.-H. Kim, S.D. Bu, T.-W. Noh, H.-J. Lin, H.-H. Hsieh, and C.T. Chen, Phys. Rev. Lett. 90, 017401 (2003).

29. S. Kumar, S. Gautam, G.W. Kim, F. Ahmed, M.S. Anwar, K.H.C.H.K. Choi, H. Chung, and B.H. Koo, Appl. Surface Sci. 257, 10557 (2011). 
30. S. Ogale, D. Kundaliya, S. Mehraeen, L. Fu, S. Zhang, A. Lussier, J. Dvorak, N. Browning, Y. Idzerda, and T. Venkatesan, Chem. Mater. 20, 1344 (2008).

31. E. Sakai, K. Amemiya, A. Chikamatsu, Y. Hirose, T. Shimada, and T. Hasegawa, J. Magn. Magn. Mater. 333, 130 (2013).

32. F. Delogu, J. Alloys Comp. 468, 22 (2009).

33. H.M. Cheng, J.M. Ma, Z.G. Zhao, and L.M. Qi, Chem. Mater. 7, 663 (1995).

34. A. Kumar, A.R. Madaria, and C.W. Zhou, J. Phys. Chem. C 114, 7787 (2010).

35. J.K. Burdett, T. Hughbanks, G.J. Miller, J.W. Richardson, and J.V. Smith, J. Am. Chem. Soc. 109, 3639 (1987).

36. V.N. Antonov, H.A. Dürr, Y. Kucherenko, L.V. Bekenov, and A.N. Yaresko, Phys. Rev. B 72, 054441 (2005).

37. V.N. Antonov, O. Jepsen, A.N. Yaresko, and A.P. Shpak, J. Appl. Phys. 100, 043711 (2006).

38. V.N. Antonov, A.N. Yaresko, and O. Jepsen, Phys. Rev. B 81, 075209 (2010).
39. O.K. Andersen, Phys. Rev. B 12, 3060 (1975).

40. V.V. Nemoshkalenko, A.E. Krasovskii, V.N. Antonov, Vl.N. Antonov, U. Fleck, H. Wonn, and P. Ziesche, Phys. Status Solidi B 120, 283 (1983).

41. J. Perdew and Y. Wang, Phys. Rev. B 45, 13244 (1992).

42. P.E. Blöchl, O. Jepsen, and O.K. Andersen, Phys. Rev. B 49, 16223 (1994).

43. J.L. Campbell and T. Parr, At. Data Nucl. Data Tables 77, 1 (2001).

44. V.I. Anisimov, J. Zaanen, and O.K. Andersen, Phys. Rev. B 44, 943 (1991).

45. A.N. Yaresko, V.N. Antonov, and P. Fulde, Phys. Rev. B 67, 155103 (2003).

46. J. Zaanen, G.A. Sawatzky, J. Fink, W. Speier, and J.C. Fuggle, Phys. Rev. B 37, 4905 (1985).

47. J. Schwitalla and H. Ebert, Phys. Rev. Lett. 80, 4586 (1998).

48. P. Krüger and C.R. Natoli, Phys. Rev. B 70, 245120 (2004).

49. A.L. Ankudinov, A.I. Nesvizhskii, and J.J. Rehr, Phys. Rev. B 67, 115120 (2003). 